INPLASY

PROTOCOL

To cite: Huang et al.

Prognostic value of CTassessed skeletal muscle in critically ill patients: a systemic review and meta-analysis. Inplasy protocol 202130031. doi:

10.37766/inplasy2021.3.0031

Received: 09 March 2021

Published: 09 March 2021

Corresponding author:

Hui-Bin Huang

hhba02922@btch.edu.cn

Author Affiliation: Department of Critical Care Medicine, Beijing Tsinghua Changgung Hospital, School of Clinical Medicine,

Tsinghua University

Support: None.

Review Stage at time of this submission: Preliminary searches.

Conflicts of interest:

None declared.

\section{Prognostic value of CT-assessed skeletal muscle in critically ill patients: a systemic review and meta- analysis}

Huang, HB1; Xu, Y2; Zheng, WH33; Zhou, H4; Zhu, Y5.

Review question / Objective: Skeletal muscle reserves are crucial to critically ill patients' recovery and survival. We aimed to investigate the prevalence of skeletal muscle loss and prognostic value of Computed tomography (CT) -assessed skeletal muscle in such patient population.

Condition being studied: The authors of the current study come from a tertiary hospital in China and all the members have extensive experience in treating critically ill patients with Malnutrition. Furthermore, these authors have published several meta-analyses, which can guarantee the completion of the current study.

Information sources: We will search the references in the included studies and personal files. We will request advice from experts in the field. Additionally, we will search associated articles from critical care, surgical, infection meetings; and contacted the authors of included trials, if need.

INPLASY registration number: This protocol was registered with the International Platform of Registered Systematic Review and Meta-Analysis Protocols (INPLASY) on 09 March 2021 and was last updated on 09 March 2021 (registration number INPLASY202130031).

\section{INTRODUCTION}

Review question / Objective: Skeletal muscle reserves are crucial to critically ill patients' recovery and survival. We aimed to investigate the prevalence of skeletal muscle loss and prognostic value of Computed tomography (CT) -assessed skeletal muscle in such patient population.

Condition being studied: The authors of the current study come from a tertiary hospital 
in China and all the members have extensive experience in treating critically ill patients with Malnutrition. Furthermore, these authors have published several meta-analyses, which can guarantee the completion of the current study.

\section{METHODS}

Participant or population: Adult ( $\geq 18$ years old) ICU patients.

Intervention: Evaluation of CT-assessed skeletal muscle with any clear and objective method.

\section{Comparator: None limitation.}

Study designs to be included: Eligible studies had a cohort, case-control or RCT design.

Eligibility criteria: We included articles evaluating the association between CTassessed skeletal muscle and critically ill patients' risk of mortality.

Information sources: We will search the references in the included studies and personal files. We will request advice from experts in the field. Additionally, we will search associated articles from critical care, surgical, infection meetings; and contacted the authors of included trials, if need.

Main outcome(s): The prevalence of CTassessed SML and its prognostic value in such a patient population.

Quality assessment / Risk of bias analysis: The Newcastle-Ottawa Scale.

Strategy of data synthesis: An overall effect estimate for all data as risk ratio (RR) / mean difference (MD) with $95 \% \mathrm{Cl}$ will be calculated. The presence of statistical heterogeneity among the studies by using the $Q$ statistics and the heterogeneity by using the 12 statistic was addressed. A pvalue of less than 0.10 or an 12 value of greater than $50 \%$ as indicative was considered of substantial heterogeneity. A random-effects model or a fixed-effects model (DerSimonian-Laird) will be chosen when significant heterogeneity or nonsignificant heterogeneity was not observed, respectively.

Subgroup analysis: 1. Geographic location: Asian and non-Asian countries; 2. Patient population: unselected critical illness, trauma, and transplantation; 3. Muscle measured: SMA/SMI, TPA/TPI, or SMD; 4. Follow-up duration: long-term or shortterm mortality; 5. The adjusted analysis used or not.

Sensitivity analysis: By excluding one study, in turn, to explore whether an individual study's particular result drove the results.

\section{Country(ies) involved: China.}

Keywords: skeletal muscle; sarcopenia; meta-analysis; critically ill patient; mortality.

Contributions of each author:

Author 1 - Hui-Bin Huang.

Author 2 - Yuan Xu.

Author 3 - Wen-He Zheng.

Author 4 - Hua Zhou.

Author 5 - Yuan Zhu. 